\title{
8 $.8 \%$ \\ Erratum: Die bedeutende nicht-proteinogene Aminosäure Norleucin und ihre komplizierte Namensfindung
}

\section{Carsten A. Vock*}

*Korrespondenz: Dr. C. A. Vock, E-Mail: carsten.vock@univie.ac.at, aDepartment für Ernährungswissenschaften, Fakultät für Lebenswissenschaften, Universität Wien, Althanstr. 14 (UZA II), A-1090 Wien; 'Fakultät für Chemie, Universität Wien, Währinger Str. 38-42, A-1090 Wien.

\section{Abstract: Inhaltliche Richtigstellung zu folgendem Beitrag in der Chemical Education-Reihe: C. A. Vock, Chimia 2021, 75, 443-445, https://doi.org/10.2533/chimia.2021.443.}

Keywords: Aminosäuren · Bioisosterie $\cdot$ Chemical Education Nomenklatur

Im Rahmen der finalen Drucklegung der Publikation ${ }^{[1]}$ wurde ein Satz ohne Wissen des Autors versehentlich geringfügig inhaltlich verändert. Die geänderte und gedruckte Version entspricht allerdings nicht mehr der Nomenklatur-Systematik, die im Artikel eigentlich vermittelt werden sollte.

Zur Richtigstellung sollte daher folgender Satz (Seite 444, linke Spalte)

„Im Bereich der Neurotransmitter und Nebennierenhormone unterscheidet sich Noradrenalin 9 von Adrenalin 8 durch das Fehlen einer $\mathrm{CH}_{3}$-Gruppe, was in diesem Fall den Unterschied zwischen einem primären Amin und einer N-Methylverbindung bedeutet. “

durch die ursprünglich vom Autor beabsichtigte Form

„Im Bereich der Neurotransmitter und Nebennierenhormone unterscheidet sich Noradrenalin 9 von Adrenalin 8 ebenfalls durch das Fehlen einer $\mathrm{CH}_{2}$-Gruppe, was in diesem Fall den Unterschied zwischen einem primären Amin und einer $N$-Methylverbindung bedeutet. “

ersetzt werden. Hierzu eine kurze Begründung:

Nach IUPAC zeigt die Vorsilbe Nor- die Entfernung eines unsubstituierten gesättigten Gerüstatoms samt der daran gebundenen Wasserstoffatome aus Ring oder Seitenkette einer entsprechenden Vorläuferverbindung an. ${ }^{[2]}$ In den allermeisten Fällen handelt es sich dabei um eine $\mathrm{CH}_{2}$-Gruppe (Methylengruppe). Der in der Chemie gängigerweise verwendete Begriff einer „fehlenden Methylgruppe“ steht eigentlich - wenn man die Summenformeln betrachtet - für den Austausch einer Methylgruppe gegen ein Wasserstoffatom, also formell ebenfalls für das Fehlen einer $\mathrm{CH}_{2}$-Einheit. Während für Chemiestudierende im Hauptfach diese unterschiedlichen Betrachtungsweisen keine größeren Verständnisprobleme hervorrufen sollten, ist es beim Chemieunterricht für Nebenfachstudierende von großer Wichtigkeit, klare und möglichst allgemeingültige Definitionen zu verwenden. Auch wenn es vielleicht „haarspalterisch“ klingen mag, empfiehlt der Autor daher, bis zum Auftreten spezieller Probleme bei der Verwendung der Vorsilbe Nor- generell vom Fehlen einer $\mathrm{CH}_{2}$-Einheit zu sprechen.

Zur Veranschaulichung ist das Entfernen der jeweiligen $\mathrm{CH}_{2}$-Einheit in Schema 1 nochmals detailliert für die bereits in der Originalpublikation gezeigten Beispiele Estradiol $\mathbf{6} \rightarrow$ B-Norestradiol A und Adrenalin $8 \rightarrow$ Noradrenalin 9 sowie zusätzlich für Progesteron $\mathbf{B} \rightarrow$ 18,19-Dinorprogesteron $\mathbf{C}^{[3]}$ dargestellt.

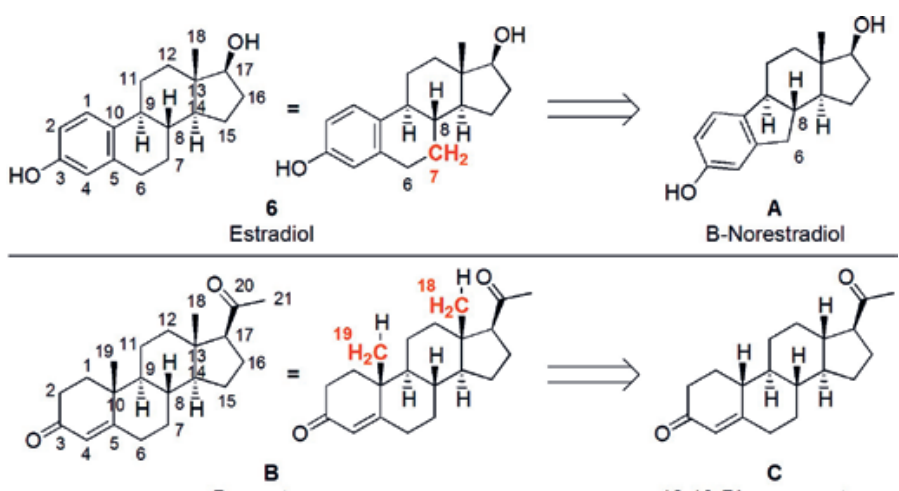

Progesteron

18,19-Dinorprogesteron<smiles>C=CC=CCCNC[C@H](O)c1ccc(O)c(O)c1</smiles>

Schema 1. Beispiele für Nor-Verbindungen und Erklärung ihrer Namensbildung durch formelle Entfernung einer oder mehrerer Methylengruppen aus den Stammverbindungen (entfernte $\mathrm{CH}_{2}$ Gruppen in rot markiert).

Eingegangen: 28. Mai, 2021

[1] C.A. Vock, Chimia, 2021, 75, 443, https://doi.org/10.2533/chimia.2021.443.

[2] H. A. Favre, W. H. Powell, 'Nomenclature of Organic Chemistry: IUPAC Recommendations and Preferred Names 2013', Royal Society of Chemistry, Cambridge, England, 2014, S. 18 (P-13.4.3.2).

[3] N. A. Nelson, R. B. Garland, J. Am. Chem. Soc. 1957, 79, 6313, https://doi.org/10.1021/ja01580a053. 